\title{
STUDY OF PRESCRIBING PATTERN AND USE OF ANTIBIOTIC IN THE MANAGEMENT OF WOUND INFECTION
}

\author{
NIKHIL PETER ${ }^{1 *}$, NISSY CHERIAN ${ }^{1}$, SAGY THOMAS ${ }^{1}$, SNEHA GEORGE ${ }^{1}$, JUNIOR SUNDRESH N ${ }^{2}$ \\ ${ }^{1}$ Department of Pharmacy Practice, Annamalai University, Chidambaram, Tamil Nadu, India. ${ }^{2}$ Department of Surgery, Rajah Muthiah \\ Medical College \& Hospital, Annamalai University, Chidambaram, Tamil Nadu, India. Email: nikhilpetersara1@gmail.com
}

Received: 20 September 2016, Revised and Accepted: 26 October 2016

\section{ABSTRACT}

Objective: The main objective of the study is to estimate the demographic details of patients with various wound infection and to observe the various organism causing infections and also to analyze the prescribing pattern of antibiotic used in wound infection.

Methods: This is a prospective observational study to evaluate the prescribing pattern of antibiotics in the Department of Surgery. The study was conducted for 4 months (April 2016-July 2016), the information was collected from the case sheets, and the data were analyzed using Microsoft Excel.

Results: In this study, males were higher than females consisting of $64.5 \%$ of the total sample size. In wound infection, diabetic foot ulcer was found to be a major problem. It can be observed that antibiotics were prescribed for all of the patients with cefotaxime being prescribed the most as monotherapy. Cefotaxime+metronidazole were commonly prescribed as dual therapy. Multivitamins, antiulcerants were other major drugs provided in the supportive care. With reference to the culture sensitivity test, the major organism isolated was Staphylococcus aureus.

Conclusion: An important consideration is needed in prescribing antibiotic therapy. The utilization of culture sensitivity studies are limited and most antibiotic selection is empirical, so proper evaluation and monitoring is needed by the health-care professionals to select the appropriate one to promote the rational use of antibiotics.

Keywords: Diabetic foot ulcer, Wound sepsis, Burns, Surgical site infection, Antibiotics.

(C) 2017 The Authors. Published by Innovare Academic Sciences Pvt Ltd. This is an open access article under the CC BY license (http://creativecommons. org/licenses/by/4. 0/) DOI: http://dx.doi.org/10.22159/ajpcr.2017.v10i2.15256

\section{INTRODUCTION}

The emergence of a resistant population of bacteria in a patient as a result of antibiotic use generally occurs through a process termed "selective pressure." Studies using special culture techniques show that healthy persons normally small numbers of bacteria that are intrinsically resistant to antibiotics [1]. When antibiotics are prescribed to large numbers of persons in a population, resistant bacteria may become the predominant organisms in that community. This situation is occurring with respiratory tract pathogens that were once universally susceptible to antibiotics [2].

The potential for infection depends on a number of patient variables such as the state of hydration, nutrition, and existing medical conditions as well as extrinsic factors, for example, related to pre-, intra-, and post-operative care if the patient has undergone surgery. This often makes it difficult to predict which wounds will become infected [3]. Consequently, the prevention of wound infection should be a primary management objective for all health-care practitioners. The main types of wound infection include burn, diabetic foot ulcer, and surgical site infection [4].

The potential pathogens of wound include bacteria, fungi, protozoa, and virus and other microorganisms which are $<0.1 \mathrm{~mm}$ in diameter and can, therefore, only be seen under a microscope. They can be categorized into different groups such as bacteria, fungi, protozoa, and viruses, depending on their structure and metabolic capabilities [5]; there are a number of ways in which microorganisms can gain access to a wound; this may consist of direct contact: Transfer from equipment or the hands of carer's airborne dispersal: Microorganisms deposited from the surrounding and selfcontamination: Physical migration from the patient's skin or gastrointestinal tract.
While there is no definitive evidence to identify the most common route of entry for a microorganism into a wound, direct contact, and poor hand-washing techniques of healthcare practitioners during pre- and post-operative phases of the patient care are considered to be significant factors.

The presence of a microorganism within the margins of a wound does not indicate that wound infection is inevitable [6]. Protective colonization may play a part whereby some bacteria produce highly specific proteins that kill or inhibit other, usually closely related, bacterial species or where certain bacteria produce a variety of metabolites and end products that inhibit the multiplication of other microorganisms [7].

The inflammatory response is a protective mechanism that aims to neutralize and destroy any toxic agents at the site of an injury and restore tissue homeostasis [8]. There are a number of indicators of infection; these include the classic signs related to the inflammatory process and further more subtle changes as highlighted by Cutting and White [9]. The classic signs of infection include localized erythema, inflammation, cellulitis, and edema.

Antibiotics are chemical substances produced by a microorganism that has the capacity, in dilute solutions, to selectively inhibit the growth of or to kill other microorganisms [4]. Whereas it is now generally accepted that systemic antibiotics are essential for the management of clinically infected wounds, the choice of antibiotic to be used is not always apparent. Only after a comprehensive assessment process including consideration of the patient characteristics, the results of microbiological investigations and the identification of both the nature and location of the wound can the most appropriate antibiotic be identified. 
The routine use of topical antibiotics is not justified for colonized or infected wounds [10]. In addition, a recent systematic review of antimicrobial agents has concluded that systemic or topical antimicrobials are not generally indicated for the management of chronic wound infections [11]. However, there may be some value in the prophylactic use of topical antimicrobials for the initial management of acute cellulites, while awaiting clarification of antibiotic sensitivity and the establishment of a therapeutic regimen. Resistance to antibiotics has become a serious problem in recent years particularly with the rise of epidemic strains of MRSA. The overuse of broad spectrum antibiotics will only serve to exacerbate the situation. It could, therefore, be argued that all antibiotic use should be based on known sensitivities.

\section{METHODS}

\section{Study site}

The study was conducted in the Department of Surgery, Rajah Muthiah Medical College. Annamalai University. A 1260 bedded multispecialty tertiary care teaching hospital.

\section{Study design}

This research is a prospective observational study.

\section{Study period and duration}

This study is conducted for 4 months period from April 2016 to July 2016.

\section{Selection procedure}

The patients admitted to the surgery wards and post-operative surgical care unit with various wound infections of burn, diabetic foot, ulcer, cellulites, and surgical wounds was selected for this study.

\section{Inclusion criteria}

- The patient who got admitted with various wound infection

- Patient with age above 18 , including both the gender

- Patient with comorbidity.

\section{Exclusion criteria}

- $\quad$ Patient who are admitted to intensive care unit

- Early discharge and treatment discontinuation.

\section{RESULTS}

In accordance with the inclusion and exclusion criteria, a total of 110 patients were selected for the study, in that males $(64.5 \%)$ outnumbered females $(35.5 \%)$. Most of the patient were found in the age group of 59-68 (23.6\%) and least were observed in the age group more than $78(8.1 \%)$ (Table 1$)$.

While considering the prescribing pattern of the patients, it is visible that all the selected patients have received antibiotic therapy. The supportive care provided to the patients includes vitamin supplements (18.5\%), analgesics (12.6\%), antiulcerant (14.2\%) and antipyretics and other medications (15.2\%) consist of antiglycemic agents, antihypertensive, and anti-emetics (Table 2).

When considering the antibiotic prescribing pattern, cephalosporin is the most frequently prescribed antibiotic (28.9\%) followed by nitroimidazole derivatives (25.1\%), and macrolides being least prescribed (2.8\%) (Table 3 ).

Culture and sensitivity pattern were performed for 22 out of 110 patients. The most common organism isolated was Staphylococcus aureus (40.9\%) followed by Pseudomonas (22.7\%) with Klebsiella being the least isolated organism (Table 4).

The patient selected had different kinds of wound with the majority of the patients belonging to diabetic foot ulcer (42), then wound sepsis (32), followed by burns (21), and lastly surgical site infections (15) (Fig. 1).
While considering the prescribing pattern of antibiotics, it is found that they were given in monotherapy, dual therapy, and triple therapy. In most of the prescription, the patient received antibiotic dual therapy which makes it the treatment of choice.

In burn cases, only one patient has received cefotaxime as monotherapy. While cefotaxime with metronidazole/gentamicin/amikacin is observed as the major combinations in dual therapy and three patients were prescribed with triple antibiotic therapy (Table 5).

Commonly used mediciation pattern in diabetic foot ulcer consists of monotherapy with cefotaxime or metronidazole. The dual therapy is usually prescribed in combinations such as cefotaxime and ceftriaxone, cefotaxime+amikacin, and ciprofloxacin+amikacin. While, the combination observed in triple therapy includes metronidazole+ofloxacin+cefixime, and amikacin+metronidazole+amo xicillin-clavulanic acid (Table 6).

Out of 32 wound sepsis, the patients four were given monotherapy with cefotaxime/ciprofloxacin. In wound sepsis cases dual therapy pattern is advised and is clearly evident as it is given to 21 patients in combinations of cefotaxime+metronidazole, cefotaxime+gentamicin, and ciprofloxacin+gentamicin. The medication regimen for triple therapy includes piperacillin-tazobactam+metronidazole+ampicillin (Table 7).

Table 1: Age wise distribution of patients

\begin{tabular}{llll}
\hline Age group & Male & Female & Total (\%) \\
\hline $18-28$ & 8 & 5 & $13(11.8)$ \\
$29-38$ & 7 & 5 & $12(10.9)$ \\
$39-48$ & 11 & 3 & $14(12.7)$ \\
$49-58$ & 9 & 8 & $17(15.4)$ \\
$59-68$ & 17 & 9 & $26(23.6)$ \\
$68-78$ & 12 & 7 & $19(17.2)$ \\
More than 78 & 7 & 2 & $9(8.1)$ \\
\hline
\end{tabular}

Table 2: Prescribing pattern of type of medication

\begin{tabular}{ll}
\hline Category & Number of drugs (\%) \\
\hline Antibiotics & $211(34.9)$ \\
Analgesics & $76(12.6)$ \\
Anti-ulcerent & $86(14.2)$ \\
Antipyretc & $26(4.3)$ \\
Vitamin supplement & $112(18.5)$ \\
Others & $92(15.2)$ \\
\hline
\end{tabular}

Table 3: Prescribing pattern of antibiotic classes

\begin{tabular}{ll}
\hline Antibiotic classes & Number of drugs (\%) \\
\hline Cephalosporins & $61(28.9)$ \\
Aminoglycosides & $32(15.1)$ \\
Fluoroquinolones & $41(19.4)$ \\
Penicillin & $18(8.5)$ \\
Macrolide & $6(2.8)$ \\
Nitro imidazole & $53(25.1)$ \\
\hline
\end{tabular}

Table 4: Distribution of organism present in culture

\begin{tabular}{ll}
\hline Organism & Number of patients (\%) \\
\hline Staphylococcus aureus & $9(40.9)$ \\
Escherichia coli & $3(13.6)$ \\
Klebsiella & $1(4.5)$ \\
Pseudomonas & $5(22.7)$ \\
Proteus mirabilis & $4(18.1)$ \\
\hline
\end{tabular}


15 patients, who were victims of surgical site infections, were prescribed with only monotherapy and dual therapy. Monotherapy with ciprofloxacin/cefotaxime and dual therapy with combinations such as cefotaxime+gentamicin and amikacin+cefotaxime (Table 8).

\section{DISCUSSION}

The use of antimicrobial agents, especially antibiotics has become a routine practice for the treatment of wound infection. In this study, out of 110 patients males (64.5\%) were found to be higher than females (35.4\%). This complies with the study conducted by Thomas [12], where males were $63.1 \%$ and similar to Immranuddin et al. [13]. Where males consist of $55 \%$ of the sample size.

Most of the patients who were included in this study belonged to the age group 59-68 (23.7\%), then $69-78(17 \%)$, and few only belongs to the age group of more than $78(8.2 \%)$. This pattern shows patients who are aged and working in the agriculture sector and are more vulnerable to get wounded, in their case, the wound healing process is slow and if not properly treated will lead to many complications.

The wound may generate in our body due to several reasons, open injuries have a potential for serious bacterial infection including gas gangrene and tetanus and this in turn may lead to long-term disabilities, chronic wound or bone infection, and death. This study undertakes major wounds consisting of diabetic foot ulcer (38\%), wound sepsis (29\%), burns (19\%), and surgical site infection (13.6\%) and this observation is similar to Thomas [12].

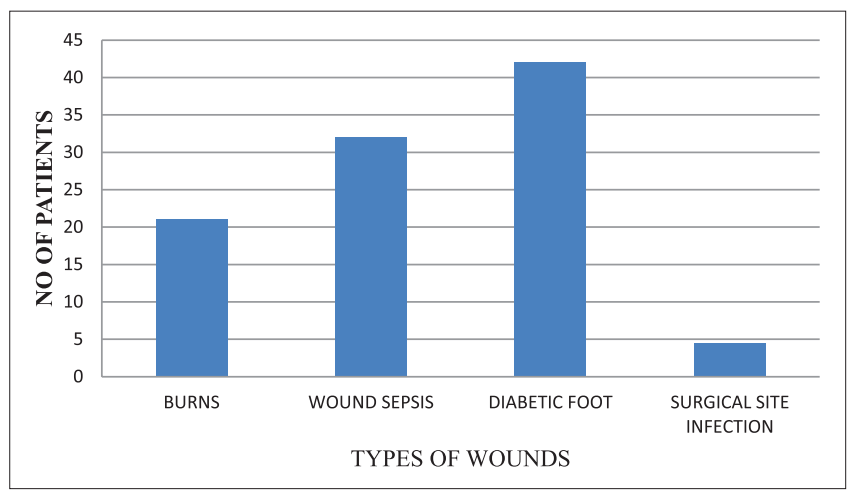

Fig. 1: Category of wound present in cases

Table 5: Prescribing pattern of antibiotic in burn

\begin{tabular}{ll}
\hline Types of therapy & Number of prescription $(\mathbf{n = 2 1 )}$ \\
\hline Mono therapy & 1 \\
Dual therapy & 17 \\
Triple therapy & 3 \\
\hline
\end{tabular}

Monotherapy: cefotaxime

Dual therapy: Cefotaxime+metronidazole, cefotaxime+gentamicin,

cefotaxime+amikacin, and ceftriaxone+erythromycin

Triple therapy: Piperacilln-tazo+metronidazole+ampicillin

\section{Table 6: Prescribing pattern of antibiotic in diabetic foot ulcer}

\begin{tabular}{ll}
\hline Types of therapy & Number of prescription \\
\hline Mono therapy & 2 \\
Dual therapy & 23 \\
Triple therapy & 17 \\
\hline
\end{tabular}

Monotherapy: Ciprofloxacin/Metronidazole

Dualtherapy: Cefotaxime+ceftriaxon, cefotaxime+amikacin,

ciprofloxacin+amikacin, metronidazole+amikacin, and

cefotaxim+metronidazole

Tripletherapy: Metronidazole+piperacillintazobactam+cefotaxime.

Amikacin+Metronidazole+amoxicillin-clavullanicacid,

cefixime+metronidazole+ofloxacin
The prescribing pattern shows antibiotics are the widely prescribed in every case irrespective of the wound type, this leads to lesser chances of secondary infections and other complications. Since most of these cases, the patients are highly prone to infections the use of antibiotics is essential in their treatment. However, prescribing antibiotics where it is not necessary can lead to resistance toward the medication. After antibiotics vitamin supplements are widely prescribed followed by antiulcerants and analgesics.

A total of 211 antibiotics for 110 patients in 1 day of the treatment course, cephalosporins are widely prescribed in surgical wards followed by nitro imidazole derivatives, the same observation was in accordance with the study carried by Immranuddin et al., [13] and the least prescribed is macrolids. Out of the organism isolated S. aureus is a Gram-positive coccal bacterium was found to be the main bacteria and Gram-negative Pseudomonas observed in five patients.

In every case, there was treatment with antibiotic monotherapy, dual therapy, and triple therapy. Such combination therapy requires an understanding of the potential for interaction between the antibiotics. Combination therapy of antibiotics can be prescribed for the treatment of polymicrobial infections, to enhance antimicrobial activity for a specific infection (i.e., for synergy) and to prevent the emergence of resistance [14]. The prescribing pattern promotes dual therapy above mono and triple therapy which is same as the study conducted by Thomas [12], in the same hospital settings with dual therapy for $45.23 \%$ of the patients.

Most of the monotherapy consist of cefotaxime or ciprofloxacin. While dual therapy consists of cefotaxime+metronidazole, followed by cefotaxime+gentamicin, and cefotaxime+amikacin which can be observed in burns, wound sepsis, diabetic foot ulcer, and surgical site infections.

Cefotaxime was the common antibiotic prescribed followed by metronidazole, the combination of cefotaxime+metronidazole is also widely prescribed as dual therapy which complies with the study by Immranuddin et al. [13].

\section{CONCLUSION}

In recent years, there has been a drive to reduce unnecessary antibiotic prescribing, which has contributed to the emergence of bacterial resistance. Antibiotics share a very high percentage in any prescription, so the prescribing pattern of antibiotic seeks evaluation and monitoring

Table 7: Prescribing pattern of antibiotic in wound sepsis

\begin{tabular}{ll}
\hline Types of therapy & Number of prescription \\
\hline Mono therapy & 4 \\
Dual therapy & 21 \\
Triple therapy & 7
\end{tabular}

Monotherapy: Cefotaxime/ciprofloxacin

Dual therapy: Cefotaxime+metronifazole, cefotaxime+gentamicin,

cefotaxime+amikacin, ciprofloxacin+gentamicin, and

ceftriaxone+metronidazole

Triple therapy: Piperaciilin-tazo+metronidazole+ampicillin,

amikacin+metro+taper

Table 8: Prescribing pattern of antibiotic in surgical site infection

\begin{tabular}{ll}
\hline Types of therapy & Number of prescription \\
\hline Mono therapy & 4 \\
Dual therapy & 11 \\
Triple therapy & 0 \\
\hline
\end{tabular}

Monotherapy: Ciprofloxacin/cefotaxime

Dual therapy: Cefotaxime+gentamicin, amikacin+cefotaxime, and metronidazole+cefotaxime 
if necessary and suggest modifications in prescribing pattern as to make medical care rational and cost-effective.

This study reveals that antibiotic selection in the hospital is not according to any standard guidelines, most of the antibiotic was prescribed empirical and only a few patients undergoes in culture sensitivity test. Improper selection and rotation of one category of antibiotic to the next before completion of minimum day, of course, may result in antibiotic resistance. The periodic study on the usage of antibiotics and sensitivity pattern in the hospital setup is to be conducted which enables the health-care professionals to select the appropriate one to promote the rational use of antibiotics.

\section{REFERENCES}

1. Olson B, Weinstein RA, Nathan C, Chamberlin W, Kabins SA. Occult Aminoglycoside resistance in Pseudomonas aeruginosa: Epidemiology and implications for therapy and control. J Infect Dis 1985;152(4):769-74.

2. Gonzales R, Bartlett JG, Besser RE, Cooper RJ, Hickner JM, Hoffman JR, et al. Principles of appropriate antibiotic use for treatment of acute respiratory tract infections in adults: Background, specific aims, and methods. Ann Emerg Med 2001;37(6):690-7.

3. Heinzelmann M, Scott M, Lam T. Factors predisposing to bacterial invasion and infection. Am J Surg 2002;183(2):179-90.
4. Recognition and Management of Wound infection. Available from: http://www.worldwidewounds.com/2004/january/collier/managementof-wound-infections.html, www.[Last accessed on 2016 Oct 17].

5. White RJ, Cooper R, Kingsley A. Wound colonization and infection: The role of topical antimicrobials. Br J Nurs 2001;10(9):563-78.

6. Brook I, Frazier EH. Aerobic and anaerobic bacteriology of wounds and cutaneous abscesses. Arch Surg 1990;125(11):1445-51.

7. Kingsley A. A proactive approach to wound infection. Nurs Stand 2001;15(30):50-4, 56, 58.

8. Collier M. Universal concepts. J Wound Care 2001;10(7):249.

9. Cutting KF, White R. Defined and refined: Criteria for identifying wound infection revisited. Br J Community Nurs 2004;9(3):S6-15.

10. Nagoba BS, Selkar SP, Mule JB. Irrigating methicillin-resistant Staphylococcus aureus-colonized and - Infected chronic wounds - Why use tap water? Int Wound J 2013;12(5):605.

11. O'Meara S, Cullum C, Majid M, Sheldon T. IR7: A systematic review of systemic and topical antimicrobial agents used in the prevention and treatment of chronic wounds. Value Health 1999;2(5):359.

12. Thomas $Z$. Study on drug utilization, prescribing pattern of antibiotic in the management of diabetic foot ulcer. IJIPSR 2015;3(8):1037-49.

13. Immranuddin M, Asfin M, Sruthi VV, Deshpande S, Kumar TP, Reddy MV, et al. cost analysis and prescribing patterns of antibiotic in post operative surgery patients. IAJPR 2015;12(11):2231-6876.

14. Dipiro JT, Talbert RL, Yee GC, Matzke GR, Wells BG, Michaelposey L. Pharmacotherapy: A Pathophysiologic Approach. $8^{\text {th }}$ ed. United States of America: McGraw-hill Companies; 2011. p. 16. 